\title{
Impact Failure of Polycrystalline Diamond Cutters
}

\author{
Robin C Laurence ${ }^{1 *}$, Richard I Todd ${ }^{1}$, and Clive R Siviour ${ }^{2}$ \\ ${ }^{1}$ Unversity of Oxford, Department of Materials, Parks Road, Oxford, OX1 3PH, UK \\ ${ }^{2}$ University of Oxford, Department of Engineering Science, Parks Road, Oxford, OX1 3PJ, UK
}

\begin{abstract}
Polycrystalline Diamond (PCD) Cutters are ultra-hard tools used for oil and gas drilling. These tools can fail in the form of spalling due to impact. In this paper, direct impact and quasi-static loading are used to investigate the failure force for leached PCD cutters when loaded at an angle on the edge of their chamfer with a PCD striker or platen. Impact experiments were performed using an impedance match system which ensures the sample is only loaded once; this allows subsequent analysis of the failure surfaces. The failure force at high deformation rate is around $8 \mathrm{kN}$ and at low deformation rate $6.3_{-1}^{+2.3} \mathrm{kN}$. The failure force required for spalling increases with increasing deformation rate. High speed imaging is also used to explore the failure history of the cutters.
\end{abstract}

\section{Introduction}

Polycrystalline Diamond (PCD) Cutters are ultra-hard tools used for oil and gas drilling. Their microstructure consists of diamond grains on the order of tens of micrometres in size and a binder consisting of cobalt-based alloy particles. The cutters are produced by sintering diamond powder on a tungsten-carbide-cobalt cermet substrate at high pressure (4-10 GPa) and high temperature $\left(1400-1800^{\circ} \mathrm{C}\right)$ [1] to form a diamond table disc of typical radius $8 \mathrm{~mm}$ and thickness $3 \mathrm{~mm}$.

In drilling, PCD cutting tools traditionally suffer from smooth wear failure. However, in some applications cutter failure in the form of spalling as a result of impact becomes more prevalent. This is attributed to an interruption in contact between the cutter and the rock surface, resulting in impacts on the cutter when the cutter regains contact with the rock surface. The work presented in this paper investigates the effect of deformation rate on the failure of PCD cutters. This is done through consideration of forces during loading events combined with high-speed imaging. Finite element analysis is also considered.

The PCD cutters used in this investigation have diameter $15.6 \mathrm{~mm}$, height $13 \mathrm{~mm}$ and diamond table thickness $3 \mathrm{~mm}$ and have a planar interface between the diamond table and the substrate. Additionally, the cutters are leached and chamfered on the top edge of the cutter at a $45^{\circ}$ angle. The leaching process uses acid to remove the binder from between the grains

* Corresponding author: Robin.laurence@materials.ox.ac.uk 
in a layer near the surface. This has the effect of reducing the fracture toughness of the PCD [2] but increases the tool life in standard smooth wear operation [3].

\section{Experimental set up and procedure}

\subsection{High deformation Rate}

To create high deformation rate failures with a single loading, a direct impact Hopkinson bar set up [4] is used, shown in Figure 1. This consists of a striker of length $226 \mathrm{~mm}$ which has PCD cutter imbedded at the end such that the diamond cutters are struck by the diamond of the projectile. The sample cutter is placed at the end of a $1 \mathrm{~m}$ steel bar angled such that the impact will occur at an angle comparable to the in-field cutting angle. Both the striker and the output bar are impedance matched to the cutter. This ensures that the cutter is only loaded a single time. Four strain gauges are placed around the centre of the output bar such that the longitudinal wave history of the impact can be recorded. This stress wave provides information as to the maximum stress supported by the cutter during the impact and can then be compared with finite element simulations to gain insight into internal stresses within the cutter during the impact event. The striker is propelled using pressurised gas to provide impact speeds ranging from 1.1 to $3.5 \mathrm{~m} \mathrm{~s}^{-1}$. The impact is recorded using high speed photography in the range of 20,000 to 100,000 frames per second; this provides an accurate impact velocity and can be compared with the strain gauge output to better understand features within the waveform.

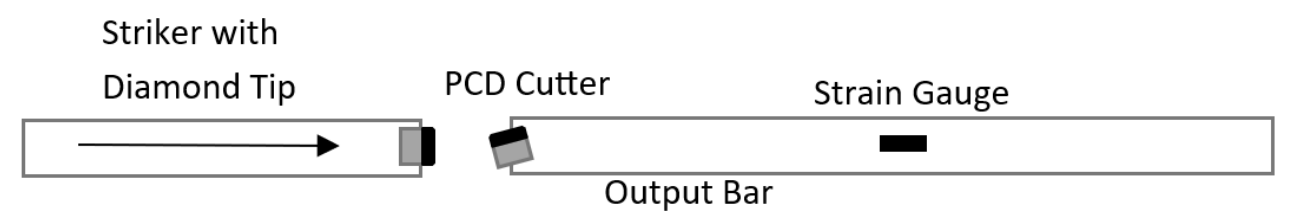

Fig. 1. Direct impact Hopkinson bar setup.

\subsection{Low deformation Rate}

The low deformation rate failures are produced under compressive loading using an Instron loading frame. This loads the cutters at a rate of $60 \mu \mathrm{m}$ per minute again using a PCD cutter imbedded in a flat platen such that the loading surface is diamond. The sample cutter is placed at an angle and loaded on the cutting edge such that the relative geometry is the same angle as the impact tests. The geometry of the setup is shown in Figure 2. The loading is filmed at five frames per second. The sample cutters are also painted with a white speckled pattern to allow Digital Image Correlation to be conducted. The load-displacement curve is recorded throughout the loading event. This provides a failure load for the cutters and can be used to observe load drops prior to failure. 


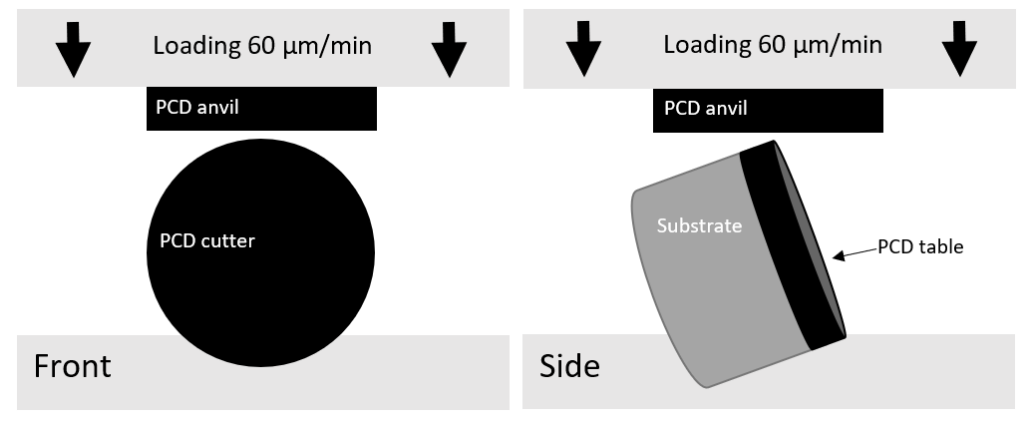

Fig. 2. Diagram of the front and side view of the quasi-static loading arrangement.

\section{Results and discussion}

\subsection{Loading force comparison}

Using the set up described in section 2.1, cutters were impacted at various speeds ranging from 1.1 to $3.5 \mathrm{~m} \mathrm{~s}^{-1}$. These impacts resulted in maximum loading forces between 6 and $15 \mathrm{kN}$. A representation of observed forces and failures is shown in Figure 3. From this the threshold failure force is assumed to be around $8 \mathrm{kN}$. At this value many cutters seem to begin to spall, though a single cutter supported a force of $8.1 \mathrm{kN}$ and did not spall.

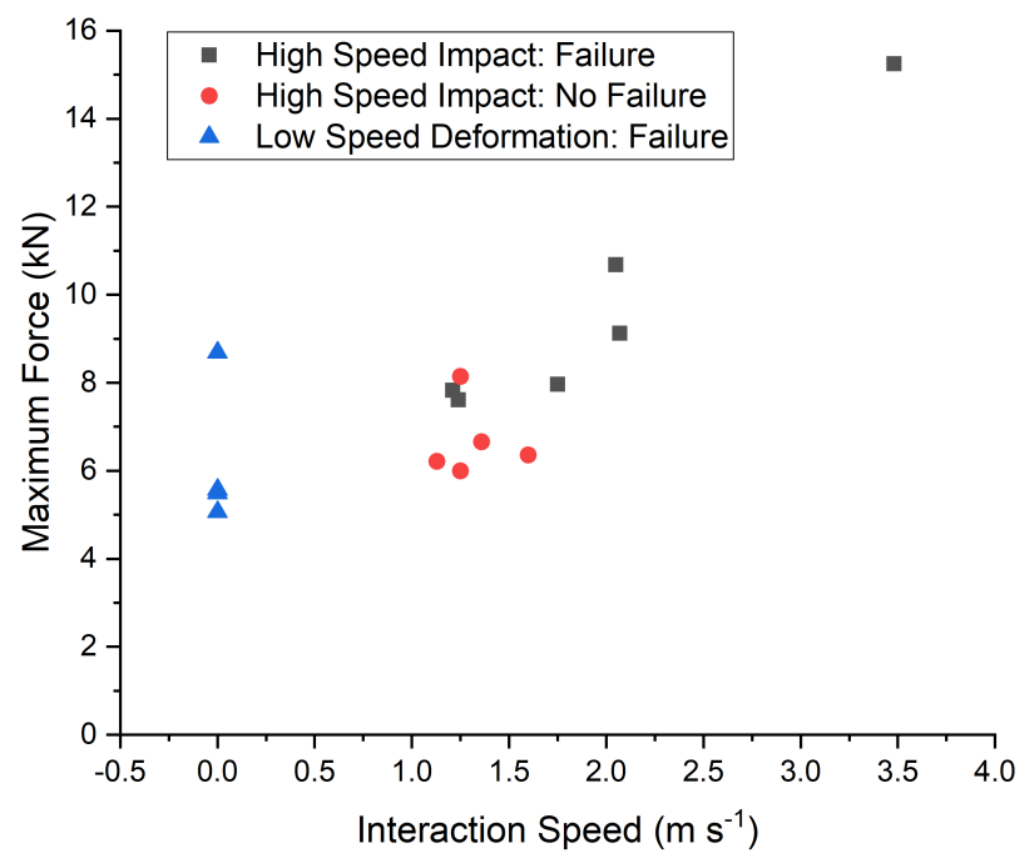

Fig. 3. Maximum force achieved for a number of cutters for both high and low deformation rate. 
Four cutters were loaded using the low deformation rate setup described in section 2.2 at a rate of $60 \mu \mathrm{m}$ per minute. From this the average failure load at $60 \mu \mathrm{m}$ per minute is found to be $6.3_{-1}^{+2.3} \mathrm{kN}$. The value is the average of the peak forces reached during loading before catastrophic fracture. The error indicates the spread in the data. The data are visually represented in Figure 3 along with the high deformation rate failure loads. The above indicates the threshold force for failure is lower at lower deformation rate, whereas McNamara et al. showed that for single edge notch beams made from a single grain size PCD cutter fracture toughness decreased with increasing deformation rate [2].

In both deformation rate regimes, some cutters outperform the normal failure limit. This is a common phenomenon in ceramics as failure of brittle materials is dependent on the size of the largest flaw within the material [5]. If a common large flaw is lacking in some samples, they will require higher forces to fail. The stress intensity factor, which dictates whether a crack will propagate is dependent on both the applied stress and the flaw size; if the largest flaw size is absent, the next largest flaw is required to instigate the catastrophic failure. As this flaw is smaller the applied stress must be larger to result in failure.

\subsection{High deformation rate impact imaging}

Figure 4A shows example stress waves from the output bar for cutters impacted at high deformation rate for speeds that result in an impact below the failure load, $1.3 \mathrm{~m} \mathrm{~s}^{-1}$, above the failure load, $2.1 \mathrm{~m} \mathrm{~s}^{-1}$, and far in excess of the failure load, $3.5 \mathrm{~m} \mathrm{~s}^{-1}$. This is then additionally compared, in Figure $4 \mathrm{~B}$, to the result of a finite element analysis of an experiment with an impact speed of $1.3 \mathrm{~m} \mathrm{~s}^{-1}$ without any fracture. For the cutter where no failure is observed, the stress wave within the output bar is sinusoidal in nature with limited high frequency activity. This is indicative of the cutter being loaded then unloaded with this wave then reflecting at the far end of the output bar. For the cutter where failure has been observed, the initial stress reached slightly exceeds that of the non-failed cutter and the loading peak is flatter containing a significant amount of high frequency oscillations. The final cutter suffered a violent catastrophic failure with the spall penetrating deep into the diamond table both vertically and horizontally, resulting in a large loss of material. When the cutter that did not fail is compared to a finite element simulation of an experiment at the same speed, Figure 4B, the stress wave is of similar shape and amplitude. The model can be used to explore the magnitudes and locations of stresses within the cutter itself during impact, which are unable to be directly probed experimentally. For this impact, for example, the maximum principal tensile stress observed anywhere within the diamond table is $1.44 \mathrm{GPa}$. This provides a bound on the fracture stress in the PCD in this geometry.

Figure 5 shows the output from the high-speed camera for the cutter which failed completely for frames corresponding to times A-D indicated in Figure 4A. Prior to the impact the sample can be seen on the left, angled as described in the experimental setup. The diamond tipped striker, on the right, impacts the diamond table on the sample at the base of the chamfer. At time A, the striker and the sample are just making contact for the first time. Between times A and B the loading begins but reaches a peak and starts to drop again. This can be seen, in the frame at time B, to be due to a spall, as is indicated by the diamond table starting to shift upwards from a point within the cutter. Time $C$ is just after the time maximum load has been achieved. As can be seen, the initial spall fragments have moved away from the impact site and a second spall is in the process of forming. Also note the sparks emitted. This indicates very high temperatures are being achieved locally to the impact. There should be limited cobalt binder within the layers of the diamond involved in the impact, as both diamond tables have been leached to remove this from the surface layer. Therefore, it is 
assumed that material producing the observed light must either be diamond or graphite; this implies that temperatures reached during the interaction may be high enough to allow either the diamond to graphite phase change or oxidation to occur. Time D indicates the end of the loading pulse. At this time, the striker stops, and the sample and output bar can separate from it. As all bars are impedance matched to the sample there should be almost complete momentum transfer. The separation can be seen in the final frame. Finally note the two-fold nature of the spall fragment. This is enlarged in Figure 6 . Here the recess left by the initial spall, light grey, can be seen within the spall fragment from the second failure. This initial spall corresponds to the load drop at time B with secondary spall occurring later in the loading. The above indicates how the high-speed photography can be used alongside the strain gauge outputs to better understand the failure history of the samples.
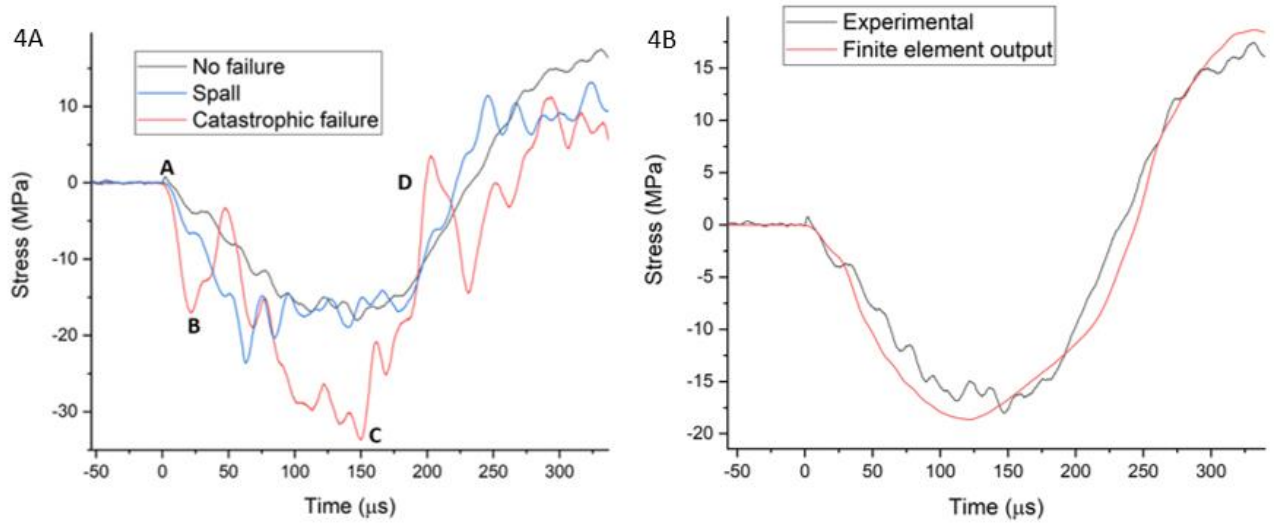

Fig. 4. A. Stress waves recorded for a number of impacts: one that did not result in failure, one that caused a spall, and one that caused catastrophic failure of the cutter. B. Stress wave produced during impact of a cutter that did not fail compared to a finite element simulation at the same speed.

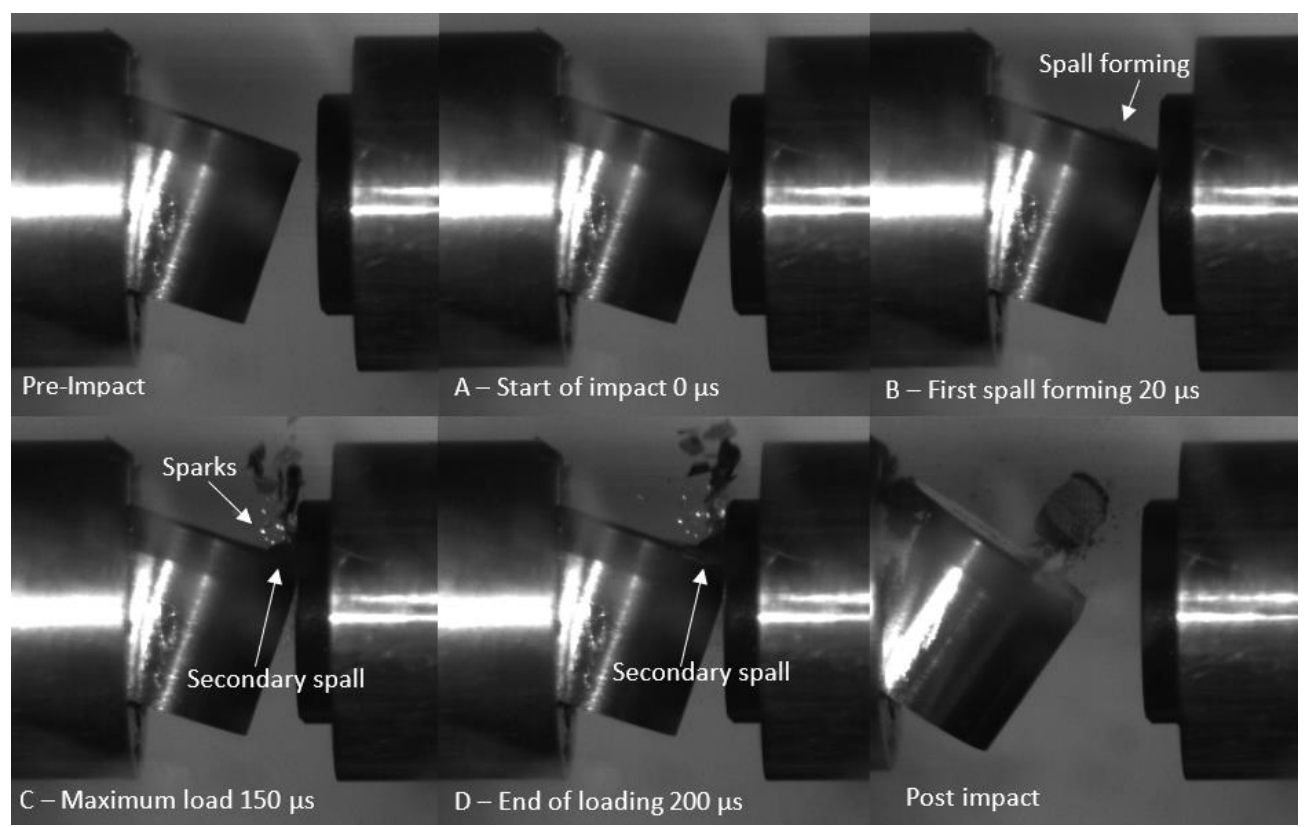

Fig. 5. Images taken during the impact of the cutter that failed catastrophically. 


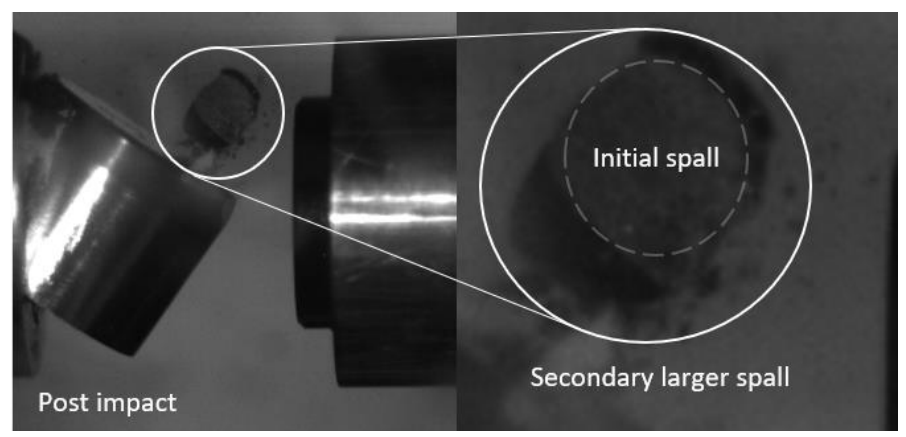

Fig. 6. Post impact image of the cutter that failed catastrophically with zoom to show both the initial and secondary spalls.

\section{Conclusions}

The effect of deformation rate on the failure force for leached PCD cutters probed with a PCD striker at an angle consistent with industrial use has been observed. At a loading rate of $6 \mu \mathrm{m}$ per minute the failure load is found to be $6.3_{-1}^{+2.3} \mathrm{kN}$ and for higher deformation rates the failure load increases to around $8 \mathrm{kN}$. High speed imaging is combined with strain gauge outputs to observe the impact history during high deformation rate experiments. In particular, the high-speed imaging confirmed the sample was only loaded once during the experiment as well as allowing correlation of the force-time traces and the observed failure events. From this, multiple spalling events can be observed in higher force impacts and it is confirmed that high temperatures are reached during impact.

\section{References}

1. T. Scott, ADV APPL CERAM, 117:3, 161-176, (2018)

2. D. McNamara, D. Carolana, P. Alveena, N. Murphya, A. Ivanković, Int J Refract Hard Met, 60, 1-10 (2016)

3. V. Astakhov and, A. Stanley. Polycrystalline Diamond (PCD) Tool Material: Emerging Applications, Problems, and Possible Solutions. Traditional Machining Processes, 1-32 (2015).

4. G. T. Gray, "Classic Split Hopkinson Pressure Bar Testing," ASM Handbook, 8, 462476, (2000)

5. B. Lawn, Fracture of Brittle Solids (2nd ed., Cambridge Solid State Science Series), Cambridge: Cambridge University Press, (1993). 